\title{
Telesecundaria: entre la teoría y la práctica
}

\section{Telesecundaria: between theory and practice}

\author{
OCHOA-VARGAS, Margarita†** \& CHÁVEZ-LÓPEZ, María Margarita \\ Centro de Investigaciones Sociales y Educativas Tecomán. Portugal \#47 Jardines del Chamizal, Tecomán, Colima.
}

ID $1^{\text {er }}$ Autor: Margarita, Ochoa-Vargas / ORC ID: 0000-0002-9652-9691

ID $1^{\text {er }}$ Coautor: María Margarita, Chávez-López / ORC ID: 0000-0002-6642-2455

DOI: $10.35429 / J O T E .2019 .7 .3 .27 .33$

Recibido 06 de Enero, 2019; Aceptado 19 de Marzo, 2019

\section{Resumen}

Este estudio surge por la necesidad de conocer los principales conflictos sobre la realidad de las prácticas docentes en educadores titulares como docentes en formación de la modalidad de Telesecundaria dentro del estado de Colima, de modo que permita el desarrollar un proyecto que contribuya a la mejora de éstas y coadyuvando el logro de los aprendizajes en los estudiantes. Es de tipo etnográfico, los informantes clave correspondían a alumnos de octavo semestre de la licenciatura en Secundaria con especialidad en Telesecundaria de la normal del estado, así como docentes en activo de las distintas zonas escolares, para sistematizar se utilizaron las técnicas propuestas por Martínez Migueles. Se encontró, que los desafíos que enfrentan en su práctica es identificar el punto de equilibrio entre la teoría y la práctica del modelo que supone la telesecundaria, la adaptación de la curricular y los programas televisivos a las necesidades reales de los alumnos, un liderazgo efectivo por parte de directores y supervisores cocientes de la realidad de las aulas así como de las características propias de cada comunidad escolar, por último, la necesidad de revalorizar la educación y la práctica del docente de telesecundaria.

Práctica docente, Revalorización de telesecundaria, Liderazgo directivo

\begin{abstract}
This study arises from the need to know the main conflicts of teaching practices in regular educators as teachers in training of the Telesecundaria modality within the state of Colima, to allow the development of a project that contributes to the improvement of these and contributes to the achievement of student learning. It is ethnographic, the key informants corresponded to eighth semester students of the Bachelor's degree in High School with a specialty in Telesecundaria of the normal state, as well as active teachers of the different school zones, to systematize the techniques proposed by Martínez Migueles were used. It was found that the challenges they face in their practice is to identify the point of balance between the theory and practice of the model that involves telesecundaria, the adaptation of the curriculum and television programs to the real needs of the students, effective leadership on the part of directors and supervisors who are aware of the reality of the classrooms as well as the characteristics of each school community. Finally, the need to revalue the education and practice of the telesecundaria teacher.
\end{abstract}

Teaching practice, Telesecundaria revaluation, Direct leadership

Citación: OCHOA-VARGAS, Margarita \& CHÁVEZ-LÓPEZ, María Margarita. Telesecundaria: entre la teoría y la práctica. Revista de Educación Técnica. 2019. 3-7: 27-33.

\footnotetext{
*Correspondencia al Autor (Correo Electrónico: cisetecoman@gmail.com)

$\dagger$ Investigador contribuyendo como primer autor.
} 


\section{Introducción}

La escuela telesecundaria tiene como sus orígenes en nuestro país en los años 60 con la intención de ampliar la educación secundaria hasta los espacios geográficos más alejados del país de modo que la sociedad mexicana recibiera alfabetización. Fue dentro del nivel de secundaria, la expresión más clara de cobertura a nivel nacional, durante el gobierno de Díaz Ordaz. En la actualidad, la telesecundarias se encuentran en el olvido del sistema educativo mexicano y con ello la práctica docente de quienes atienden esta modalidad, muestra de ello, es la poca información encontrada apara efectos del presente. Ahora bien, este es el primer acercamiento con los docentes titulares y en formación del estado de Colima, con lo que refiere a su quehacer cotidiano dentro del aula y los resultados que con ella se reflejan.

Durante el transcurso de la historia, se ha observado el ir y venir de reformas educativas en pro de la educación con la expectativa que la sociedad mexicana tenga una calidad de vida digna y a su vez, atienda las nuevas demandas de la sociedad global en la que se vive. Ciertamente cada una de ellas ha privilegiado a las reformas en las políticas públicas que ponen al frente a la educación conocida como obligatoria (preescolar, primaria, secundaria, media superior y actualmente educación inicial). Sin embargo los cambios suceden inmediatamente en preescolar, primaria y secundaria, haciendo los pilotajes correspondientes para cada nivel. En éste último, dentro de la modalidad de telesecundaria, los cambios se han dado de manera lenta sin tomar en cuenta cada una de las implicaciones que conlleva el modelo mencionado.

Una de las características principales de la modalidad de Telesecundaria es que se cuenta con un solo educador por grupo, o bien, un docente para todas las asignaturas y para los tres grados. Él es el encargado de coordinar las propuestas de trabajo de cada asignatura, mismas que están consignadas tanto en los materiales de apoyo impreso como en los programas de televisión. Otras de sus características es que atiende las zonas rurales o vulnerables, es decir en zonas suburbanas, atienden a grupos muy reducidos, esto está provocando que desaparezcan grupos o secundarias de esta modalidad completas.
Ser docente de telesecundaria implica las mismas demandas que para otra modalidad, en Colima, la escuela normal del estado oferta la Licenciatura en Educación Secundaria con especialidad en Telesecundaria, ello, permitió analizar cómo es concebida la ésta desde la escuela formadora de docentes y cómo ha sido la preparación de los alumnos próximos a incorporarse a la realidad de la escuela telesecundaria.

Ambos escenarios permitieron identificar las áreas con mayor oportunidad de esta modalidad de modo que se formularan las siguientes preguntas. ¿Cuáles son las principales dificultades que enfrentan los docentes de la modalidad de telesecundarias frente a grupo?, ¿Qué orientación reciben los docentes en formación para el trabajo en la realidad de las aulas de telesecundaria?,¿Cómo relacionan la teoría establecida en la normatividad con su práctica docente? ¿Cuáles son las dificultades principales que enfrentan los docentes frente a grupo en telesecundaria?

La investigación tuvo como propósito conocer los principales conflictos sobre la realidad de las prácticas docentes en educadores titulares como docentes en formación de la modalidad de Telesecundaria dentro del estado de Colima, de modo que permita el desarrollar un proyecto que contribuya a la mejora de éstas y coadyuvando el logro de los aprendizajes en los estudiantes

Se estructuró en tres apartados $\mathrm{La}$ realidad de las escuelas telesecundarias del estado de Colima, donde se aborda desde la matricula dentro del estado, así como el desarrollo de la metodología empleada y la extracción de las unidades de análisis, así como las técnicas e instrumentos aplicados. En el segundo apartado, Algunas de las realidades telesecundarias de las telesecundarias en México, se aborda el análisis de bibliografía sobre investigaciones realizadas en el país, que hacen referencia a las prácticas dentro del aula y las características de éstas. Por último apartado abordan las conclusiones, donde se platea lo encontrado y el cumplimiento del propósito, así como las nuevas vetas de investigación. 


\section{La realidad de las escuelas telesecundarias del estado de Colima}

El estado de Colima se caracteriza por ser una entidad federativa con poco espacio geográfico, apenas representa el $0.2 \%$ del territorio nacional según el INEGI (2019), el 11\% de la población vive en zonas rurales, es para este sector y en su ubicación donde se localizan las escuelas telesecundarias del estado. La educación telesecundaria llegó al estado de Colima en 1982 con la intención de atender a la sociedad alejada y de difícil acceso. Actualmente se cuentan con 59 telesecundarias en el estado de las cuales 26 son federales y 33 estatales, para efecto de la investigación se centró en las escuelas federales.

Según las estadísticas de la Secretaría Educación Colima, hasta el ciclo escolar 20182019 las secundarias federalizadas en la entidad tenían una matrícula de 1314 alumnos en la modalidad de secundaria, a su vez, laboran 81 docentes frente a grupo en 3 zonas escolares con las que se cuentan.

Para lleva a cabo este estudio, se entrevistaron a 15 alumnos de la escuela normal del estado de la generación 2016-2019 de un grupo constituido por 25 estudiantes y 20 docentes activos propios de la modalidad. Es un estudio de tipo exploratorio con una prueba no probabilística, pues es el primer escenario para conocer la realidad de las prácticas docentes en la aulas de las telesecundarias del estado. Para ello, se utilizaron como técnicas la observación, la entrevista; el cuestionario abierto y el análisis de documentos como lo propone Martínez Miguélez ya que es una investigación de corte cualitativo con un enfoque etnográfico.

En un primer momento, se realizó la observación directa en algunos planteles educativos para conocer la realidad de las escuelas, seguido de un análisis documental a nivel nacional. La observación realizada en los planteles se realizó en tres momentos, en el primero de ellos, se identificaron a quienes podrían ser los informantes claves, en el segundo momento se aplicó un cuestionario de preguntas abiertas a los informantes, cabe recordar que los informantes fueron tanto los docentes activos como los alumnos de octavo semestre ya mencionados, en el tercer momento, se contrastó lo que reportó el cuestionario con la realidad de las escuelas.
En el primer análisis de la información recogida se encontraron las siguientes unidades de análisis, 1) las planificaciones de los docentes en formación de telesecundaria distaban del programa vigente, 2) los docentes titulares utilizaban los programas de secundaria regular para planificar, sin embargo programaban la red de televisión con videos del programa 2006 o 2018 cuando en realidad trabajan con el plan de estudios 2011, 3) Los tiempos efectivos en clase son cortos. 4) los directores no se involucran en el trabajo académico del aula, 5) el trabajo colaborativo en las escuelas se ve sesgado por el poco liderazgo de directores y supervisores, 6) existe una mayor empatía entre los docentes con los alumnos en relación a la secundaria regular, 7) las condiciones físicas de las escuela son inapropiadas para la modalidad, pues en lagunas de ellas las televisiones o antenas no funcionan o bien, no cuentan con ellas; 8) los docentes en formación así como los titulares se sienten excluidos en las capacitaciones, 9) los docentes titulares expresan que su principal problema para trabajar son sus autoridades pues los vicios en la modalidad, pues truncan las propuestas que ellos tiene para trabajar en las aula, 10) los docentes expresan que el apoyo que más necesitan es la confianza en su trabajo, ser tomados en cuenta como las secundarias generales pues a ellos difícilmente llegan las observaciones e invitaciones a capacitaciones, 11) tanto docentes titulares como en formación sostienen su gusto por la modalidad, 12) de 81 docentes que están reportados como activos y titulares sólo 34 son especialistas en la modalidad, 13) el apoyo principal que solicitan es la capacitación de su práctica acorde a la modalidad y la evaluación de los alumnos, 14) falta de interés de los alumnos e inasistencia. 
Dichas premisas permitieron la construcción de un guion de observación que permitió orientar el tercer momento donde se focalizó la atención en la unidades de análisis mencionadas con anterioridad, donde se pudo encontrar 1) que los directivos poseen bajo resguardo material recibido por los diversos programas nuevo y bajo su resguardo, mismo al que docentes y alumnos no tiene acceso, 2) los docentes presentan su planificación mas no la ejecutan en el aula, se apoyan en los productos finales o aprendizajes esperados para solicitar los productos de los alumnos y orientar las clases, 3) la información que se baja por parte de dirección y supervisión a los docentes esta fuera de tiempo lo que deriva en que los maestros simulen los procesos, 4) la mayoría de los docentes con mayor años de servicio atienden únicamente las asignaturas que dominan de igual forma quienes tiene una perfil distinto a la modalidad, 5) la postura de los directivos impide el trabajo colaborativo entre docentes y se remiten únicamente a los administrativo, 6) las capacitaciones y Consejos Técnicos Escolares carecen de tiempo efectivo para el trabajo de las necesidades de las escuelas y docentes, 7) el trabajo con la programación en televisión es imposible debido a que de 26 escuelas que posee la modalidad en Colima solo 9 de ellas tiene los equipos completos y funcionales, 8) la inasistencia constante de los alumnos, 9) hay docentes que pese a las dificultades externas logran con éxito su práctica y el logro de los aprendizajes, son dinámicos y su índice de inasistencias por los alumnos es bajo.

Los docentes en formación por su parte, fueron entrevistados bajo los mismos indicadores que los titulares, las modificaciones en su cuestionario versaron sobre el modelo de telesecundaria que les presentan dentro de la normal y la realidad a la que se enfrentan en las aulas. Con ellos, se trabajó en los mismos tres momentos sin embargo se agregaron cuestionamientos como ¿qué relación existe de lo que supone debe ser la telesecundaria y lo que vives en tus practicas?, la respuestas de los estudiantes versaron sobre la diferencias del deber ser y lo que hacen pues afirman sus planificaciones son para cumplir con los requerimientos de la institución, pues al llegar con los titulares y al centro de trabajo, la realidad cambia y deben hacer cuanta modificación sea necesaria para atender a los alumnos.
Cuando los estudiantes normalistas deben planificar se cuestionan el porqué de no haber un programa exclusivo para la modalidad, pues las indicaciones que siguen por quien imparte el curso de práctica docente es planificar con los programas de secundaria general, de ahí que deben conseguir los programas de cada especialidad, a lo que externan le dificulta más dicho proceso. Sin embargo, pese a su esfuerzo deben adaptarse a las condiciones que se enfrenten cuando regresan al aula, durante los proceso de revisión de los docentes de observación de la practica suelen tener conflictos por estar ejecutando actividades que no están reportadas en su planificación.

Con base a la situación de no contar con un programa propio, los estudiantes comentaron que la modalidad está olvidada, en una de las clases de los docentes en formación en su escuela normal expresaron las inquietudes por no tener programas propios ni capacitaciones destinadas a la modalidad de telesecundaria, pues semanas anteriores a la clase se convocó a los alumnos de secundaria a curso de evaluación, sin embargo era destinado a secundaria general.

Uno de los puntos entre titulares y docentes en formación que expresan sesga aún más su práctica es la falta de competencia que sienten para abordar los distintos contenidos, pues consideran que la educación recibida en las escuelas normales carece del dominio en la modalidad, puesto que de los ocho semestres cursados solo tuvieron 2 que conociera la modalidad y estancia en la normal fue de un semestre. 


\section{Algunas realidades de las telesecundarias en México}

En cuanto a la revisión de documentos realizados para esta investigación, ésta tuvo como objetivo conocer las prácticas en el aula dentro de la nación mexicana bajo las unidades de análisis arrojadas en los distintos momento de las visitas a los planteles, para tener una visión más amplia de cómo se trabaja en otros espacios así como para saber qué se dice de la modalidad en el país, en ese análisis, Kalman \& Carvajal Cantillo, (2007) sostienen que la comunicación que el docente de telesecundaria establece con los alumnos es unidireccional, pues expresan que el alumno al cuestionar al maestro acepta la respuesta de éste sin dudar, trayendo con ello la marginación del conocimiento de los alumnos pues se limitan a las secuencias establecidas en los libros para el alumno o bien a las actividades que el docente consideró pertinentes por falta de tiempo.

Por otro lado, (Santos, 2001) cometa que la carga de administrativa de los directores comisionados con grupo en esta modalidad de telesecundaria incrementa la falta de supervisión por parte de las autoridades, pues al ser el docente de grupo el mismo que dirige la escuela la demanda de trámites administrativos crece, poniendo en segundo término la calidad de enseñanza del docente y deja entre dicho el logro de los aprendizajes de los alumnos. De la misma forma menciona, que la lejana de cada una de las escuelas provoca un cambio constante de maestros, pues la distancia entre estos y las familias provocan una movilidad continua a lo largo del ciclo escolar y con mayor frecuencia en las comunidades más alejadas.

Ahora bien, un estudio realizado por Educativas, (2003) sostiene que una de las virtudes de telesecundaria que favorecen el aprendizaje de los alumnos es que un solo maestro es el encargado de dirigir el aprendizaje de los alumnos, en cuestión a ello, debería suponerse que las escuelas telesecundarias lograran posicionarse en los mejores niveles de aprovechamiento del nivel, pues en dicha investigación se sostiene que el hecho de un maestro único disminuye el esfuerzo adaptativo de los alumnos permitiéndoles concentrarse en mayor medida en sus actividades escolares.
En dicho estudio Quiroz quien lo dirigió, concluyó que los alumnos de escuelas telesecundarias suburbanas logran tener seguridad por su centro escolar y un sentido de pertenencia a él, afirma que el modelo de telesecundaria podría mejorar sin embargo así como es, logra educación de calidad.

Sin embargo, Calixto Flores \& Rebollar Albarrán, (2007) menciona que las prácticas de telesecundaria están aún mu viciadas pues sostiene que los docentes aún continúan con las practicas tradicionalistas, asimismo, mencionan que las prácticas dentro de las aula de telesecundaria distan de lo que la modalidad plantea, así pues, manifiestan que el docente debe buscar formas novedosas de trabajar las tecnologías dentro de su quehacer.

Como es claro, cada autor citado con anterioridad describe parte de la realidad de las aulas de la modalidad de telesecundaria, eso permite analizar que las dificultades dentro del quehacer docente no son exclusivas de las escuelas de Colima.

A manera de conclusión, tomando como referencia lo expuesto por los autores así como las unidades de análisis obtenidas, se puede reflexionar sobre tres puntos a rescatar, 1) las prácticas en telesecundaria siguen siendo las mismas, tradicionales donde los docentes simulan la mayoría de las veces sobre cómo debe trabajarse la modalidad y lo que hacen, 2) los directores se centran en parte administrativa más no en la académica, dejando que sea el docente quien se encargue exclusivamente de las prácticas dentro del aula, 3) el modelo como tal de telesecundaria es bueno, puesto que es un solo docente, sin embargo, tanto docentes en formación como titulares carecen del dominio de la modalidad y desconocen el modelo como tal a profundidad, 4) son las prácticas viciadas dentro de telesecundaria desde la supervisión hasta el aula que provocan que los tiempos efectivos en clase sean pocos, así como que no existen innovación para el uso de la tecnología en las comunidades. 


\section{Conclusiones}

Este trabajo es la primera parte del acercamiento con las secundarias con modalidad de telesecundaria en el estado de Colima, que tenía como propósito conocer las dificultades de las prácticas de los docentes de la modalidad para diseñar un proyecto que coadyuvara al aprendizaje de los alumnos, fue de corte etnográfico, como acercamiento y elaboración de un diagnóstico de una realidad tangible tanto de las aulas en Colima como de los docentes en formación de la modalidad tratada, donde se pudo rescatar los siguiente. Existe un problema real entre la teoría y la práctica de docentes titulares como en formación.

Los primeros, presentan dificultad para llevar al aula las propuestas por el modelo, es decir, trabajar con tecnología en las zonas rurales se torna difícil debido al mal funcionamiento o inexistente material con el que cuentan, por ser una modalidad donde es un docente quien imparte todas las asignaturas, carecen del dominio de algunas, por lo que tienden a profundizar en aquellas que más les gusta o dominan. No traban con todas las asignaturas, las más rezagadas son Inglés, Educación Artística, Educación Física e Historia.

De igual forma, los titulares demandan la atención de las autoridades para que se les oferten cursos propios a la modalidad, y que las información sobre estos se de en tiempo y forma.

Demandan, que la supervisión y la dirección así como quienes cumplen la función en cada una, se involucren en la vida dentro de las aulas, pues aquellos que intentan innovar o transformar la realidad de su práctica son segados por la visión tradicionalista de sus autoridades educativas.

Eso conlleva a que el director ejerza un verdadero liderazgo al igual que el supervisor, pues ambos bajo la función que ejercen deben dirigir de manera cabal las necesidades de su escuelas, docentes, comunidad escolar a través de propiciar las situaciones necesarias para que los docentes cumplan con su función.
Por su parte, los docentes en formación señalan, que existe una brecha considerable entre la teoría que se expone en las distintas lecturas abordadas durante su paso en la normal, que éstas se encuentran desfasadas y que tanto las lectura como la normatividades establecidas para el trabajo en secundaria distan bastante con las características de las realidades en telesecundaria.

Enfatizan, que a pesar de estar por egresar, sus dudas mayores se centran en la planificación de las actividades y en el cómo evaluarlas, pues se les ha enseñado a hacerlo como en secundaria regular, mas argumentan que los problemas que enfrentan en sus escuelas son diferentes a los de una secundaria regular.

Por otro lado, la falta de material, de señal de internet, de antenas y de televisiones imposibilita a las zonas más alejadas, esto orilla a que el docente trabaje con lo que tiene, abandonado los ideales del modelo, esto indica una falla en el sistema que los docente tratan de resolver, sin embargo bajo las condiciones que presentan y la poca supervisión de las autoridades va provocando que el docente pierda interés y llene su práctica de vicios.

Los principales vicios que enfrentan las escuelas telesecundarias son la falta de tiempos efectivos en clase, pues los recesos o actividades propias de los docentes se extienden más de los regularmente permitido, de las 12 escuelas visitadas 8 extendieron su receso a una hora o más. Los CTE observados dedicaron la mayor parte del tiempo a la convivencia y a información sindical, desafortunadamente se discute muy poco lo académico.

Pese a todo lo anterior, también se observó que hay docentes comprometidos y directores que buscan dar servicio de calidad, participando en la innovación de las practicas dentro de las escuelas, los maestro que presentaron ese interés por avanzar, recibían apoyo y orientación de su director, buscaba la forma de atender las necesidades de los estudiantes y las propias partir del trabajo colaborativo. 
Con base en los anterior y tratando de cumplir el propósito de este trabajo, se establecieron los siguientes criterios que orientarán el proyecto para favorecer la práctica entre los docentes de telesecundaria, cabe señalar que el proyecto está orientado a la aplicación de la investigación-acción en las aulas, pues para atender las distintas necesidades que se enfrentan ésta metodología ofrece la transformación de la práctica y de las realidades. Si bien es cierto, telesecundaria necesita ser revalorizada pues laborar en las zonas rurales o vulnerables con adolescentes que tiene como prioridad la sobrevivencia más que la educación, no es una tarea fácil, es necesario se conozca que la modalidad de telesecundaria tiene la bondad de poder transformar la adversidad de la marginalidad de esas zonas a partir de una práctica crítica por parte de los profesores y de ellos hacia afuera.

La modalidad de telesecundaria es la posibilidad de mejorar la calidad en la educación, pues supone el uso de la tecnología en esta era tan demandante pues es explotar al máximo las habilidades natas de los alumnos. Por ello que la propuesta para el proyecto con los docentes bajo la metodología mencionada pretende, como primera acción, la capacitación de los docentes bajos las temáticas:* planeación efectiva para las escuelas telesecundarias, * evaluación en la modalidad de telesecundaria, * innovación como escuelas telesecundarias, tecnología al alcance y *reforma en el modelo de telesecundaria. Lo anterior es solo el inicio del diseño de un plan de acción de una investigación vigente.

\section{Referencias}

Calixto Flores, R., \& Rebollar Albarrán, A. (2007). La Telesecundaria, ante la sociedad del conocimiento. Revista Iberoamericana de Educación.

Educativas, I. (2003). Telesecundaria: los estudiantes y los sentidos que atribuyen a algunos elementos del modelo pedagógico. (Spanish). Revista Mexicana de Investigación Educativa.

Kalman, J., \& Carvajal Cantillo, E. (2007). Hacia una contextualización de la enseñanza y el aprendizaje en las aulas de la Telesecundaria. Revista Latinoamericana de Estudios Educativos.
Santos, A. (2001). Operación de la Telesecundaria en zonas rurales marginadas de México. Revista Latinoamericana de Estudios Educativos. 